\title{
Análisis comparativo de la divulgación corporativa en sitios web en una muestra de empresas cotizadas del mercado peruano y chileno
}

\section{Fernando Morales-Parada, Reinier Höllander-Sanhueza, Camila Varela-Castro}

\begin{abstract}
Departamento de Administración y Auditoría. Universidad del Bío-Bío Departamento de Administración y Auditoría. Universidad del Bío-Bío Departamento de Administración y Auditoría. Universidad del Bío-Bío
\end{abstract}

Con el avance de la tecnología y la enorme masificación de Internet, el uso de esa plataforma se ha vuelto un elemento clave para el mercado financiero y otros usuarios de información financiera y no financiera. El objetivo de este trabajo es determinar el grado de divulgación de información corporativa en sus sitios webs corporativos a partir de una muestra de empresas que cotizan en los mercados de valores de Chile y Perú. La muestra de investigación está compuesta por 63 compañías - 33 empresas chilenas y 30 peruanas - , que, a pesar de diferencias en las bolsas de estos países, se constituyen como las empresas cotizadas más representativas. Para ello, se configuraron 48 variables distribuidas en 5 dimensiones — rendición de cuentas, compañía y estrategia, análisis financiero, transparencia y funcionalidad- y la evaluación se mide a través de un índice. Los resultados demuestran preocupación por presentar información financiera; sin embargo, no revelan de manera significativa aspectos voluntarios sobre responsabilidad social y ambiental.

Palabras clave: Accountability, divulgaciones, reportes y transparencia, Chile y Perú.

\section{Comparative analysis of corporate disclosure in websites in a sample of listed companies in the Peruvian and Chilean market}

Since the advance of technology and the massification of Internet, the use of this platform has become a key element for the financial market and other users of financial and non-financial information. The objective of this paper is to determine the degree of disclosure of corporate information on their corporate websites studying a sample of companies listed on the Chilean and Peruvian securities markets. The research analyzes a sample composed by 63 companies, 33 Chilean companies and 30 Peruvian companies, using 48 variables distributed in 5 dimensions: Accountability, Company and Strategy, Financial Analysis, Transparency and Usability. The evaluation is measured through an index. The results show concern for presenting financial information; however, they do not reveal significant voluntary aspects, such as those related to social and environmental responsibility.

Keywords: Accountability, disclosures, reporting, transparency, Chile, Perú,

\section{Análise comparativa da divulgaçáo corporativa em websites em uma amostra de empresas listadas no mer- cado peruano e chileno}

Com o avanço da tecnologia e a enorme massa da internet, o uso desta plataforma tornou-se um elemento chave para o mercado financeiro e demais usuários de informaçóes financeiras e não financeiras. O objetivo deste trabalho é determinar o grau de divulgação de informaçóes corporativas feitas por uma amostra de empresas listadas nos mercados 
de valores mobiliários chileno e peruano, em seus sites corporativos. A amostra da pesquisa é baseada em 63 empresas, 33 empresas chilenas e 30 empresas peruanas. Para tanto, foram montadas 48 variáveis distribuídas em cinco dimensões: Accountability, Empresa e Estratégia, Análise Financeira, Transparência e Funcionalidade e a avaliação é mensurada por meio de um índice. Os resultados mostram preocupação em apresentar informações financeiras, porém, não revelam de forma significativa aspectos voluntários de responsabilidade social e ambiental.

Palavras-chave: Accountability, divulgações, relatórios e transparencia, Chile, Peru,

\section{Introducción}

El fenómeno de Internet como medio de divulgación corporativa sucedió en épocas en que la discusión contable se centraba en la necesidad de uniformar los criterios contables entre los países. Un débil proyecto surgido en la década de 1970 con la creación del IASC, e importantes análisis y discusiones al interior de la Unión Europea junto con otros organismos de nivel mundial interesados en el asunto evidenciaron la diversidad contable internacional existente. En paralelo, hubo promisorios estudios de investigación que fueron configurando un escenario proclive a la búsqueda de soluciones contingentes. En este sentido, uno de los elementos discutidos a lo largo del tiempo ha sido la valorización de intangibles, el reconocimiento, y la representación del capital intelectual y los valores razonables, entre otros. Todos son parcialmente aceptados desde las IFRS, pero con las limitaciones propias que el marco conceptual define, y que cada norma específicamente restringe o acepta. En este sentido, las empresas en general buscan otras salidas para proveer demostraciones que quizá se mantienen ajenas a los reportes tradicionales. En este punto, Internet desempeña un rol clave en los mercados.
Desde un incipiente inicio de los años 2000 hasta la actualidad, las webs pueden hacer lo mismo que en los medios impresos y algo más: pueden dar a conocer los productos y servicios de la empresa, enviar información de referencia sobre lo que se ofrece y comunicar la imagen de la empresa de forma más gráfica que el correo electrónico (Pablo, 2006). Es decir, Internet es un medio de comunicación más, pero con las ventajas propias de la tecnología sin perder la esencia de informar y proyectar a la compañía a su entorno. Esta idea está más desarrollada por AECA (2004), que observa el crecimiento de Internet como medio para distribuir información financiera y no financiera ${ }^{1}$. A partir de ello, ha modificado la forma en que la información fluye hacia los inversores, acreedores y otros usuarios externos. De este modo, la web representa un entorno completamente nuevo de difusión de información financiera.

Según Giner (1995), la información financiera divulgada por las empresas puede considerarse como una señal: no solo la información en sí misma puede tener este carácter, sino la forma de darla; los cambios; e, incluso, la ausencia de información. Es decir, se ha unido una necesidad de uniformidad informativa

\footnotetext{
1 La información no financiera es entendida como «aquella que no está reflejada en los estados financieros tradicionales, pudiendo comprender: información revelada con carácter obligatorio (informe de auditoría; hechos relevantes) e información de carácter voluntario (comentario de directivos)» (FASB, 2001). Asimismo, FASB añade que «cualquier medida no financiera, debería ser capturar los datos que informen sobre aquellos aspectos del negocio, que no estén reflejado en los estados financieros básicos» (2001).
} 
contable mundial con un incremento de divulgación no financiera. Ambos aspectos se han canalizados a través de Internet, principalmente por los sitios web de las propias compañías; los sitios de reguladores; y, también, en las redes sociales (como, por ejemplo, Twitter), que ya no solo se utilizan para efectos comerciales o relacionales, sino también para enviar señales al mercado y lograr reputación corporativa.

\section{Marco teórico}

Iniciado el siglo XXI, las tecnologías de la información y comunicación (TIC) están transformando el canal y los modelos de comunicación entre las sociedades cotizadas y cualquier agente interesado (stakeholders) en la evolución de su negocio (Gandía y Pérez, 2005). Este panorama es consistente con el hecho de que alrededor del mundo se ha incrementado el número de empresas que tienen sitios de Internet en la World Wide Web. Paralelamente, el rápido incremento del uso de la web para la divulgación financiera y de negocios ha implicado un incremento de las inversiones online (Lymer et al., 1999).

Así, Lymer et al. (1999) presentó su discussion paper "Business reporting on the Internet», a partir del cual se comenzó a sentar las bases de una materia hasta entonces poco investigada. Se trata de analizar y promover los efectos sobre una comunidad de usuarios de información financiera divulgada en Internet, además de promover la armonización contable a través de las NIIF que este mismo organismo emite. En dicho documento, el organismo internacional concluyó tres aspectos que deberían desarrollarse: a) establecer estrategias a revisar en los países con respecto del impacto del reporting ${ }^{2}$ en Internet, b) desarrollar un marco conceptual sobre el tema y c) formar comisiones de investigación que aporten sobre el tema.

A juicio de Gandía (2001), Internet posee tres condiciones que lo posicionan fuertemente en materias de divulgación de información empresarial. En primer lugar, no se trata de un único medio de comunicación, sino más bien un conjunto de medios que, utilizando un mismo canal físico de transporte, ofrece distintas alternativas. En segundo lugar, es el primer medio de comunicación de masas bidireccional, en que existe interactividad real entre emisor y receptor de la información. Finalmente, crece y se transforma con rapidez.

En este contexto, AECA (2002) entiende que los avances tecnológicos en materia de procesamiento y transmisión de la información tienen su máxima expresión en la digitalización de la información y el uso de Internet como canal de comunicación idóneo para su propagación. El efecto de masificación de esta plataforma ha sido aprovechado por la empresa como medio de divulgación. Al respecto, Serrano et al. (2003) sostienen que cada vez más las empresas divulgan información financiera en Internet frente a la rigidez, monotonía y limitaciones de la información financiera en papel. El World Wide Web ofrece fabulosas posibilidades multimedia para presentar los datos, capacidad para llegar a todos los públicos, y acceso a un mayor volumen y profundidad de la información.

Latinoamérica entró después que Europa y otros países desarrollados en armonización internacional, lo que ha implicado un largo camino con ajustes en diferentes ámbitos y a ritmos diversos entes los países latinoamericanos. Morales y Jarne (2006) evidenciaban que la región no ha sido ajena a la búsqueda de soluciones al problema de la diversidad contable internacional.

\footnotetext{
2 Reporting es un anglicismo utilizado para referirse a las divulgaciones o revelaciones corporativas realizada.
} 
Los autores destacan el rol y la temprana discusión al interior del Mercosur y el decidido respaldo del BID -que apoyó a los países en vías de desarrollo entre los años 2007 a 2011-, lo que permitió la adopción de algunos países y el establecimiento de planes de convergencia. Sin embargo, se observa un retraso respecto al contexto europeo, puesto que, mientras se estaba en convergencia, se avanzó fuertemente en estándares para el reporting en Internet, mientras que, en el contexto latinoamericano, se avanzó con rezago en lo primero (convergencia contable mundial) y no hay evidencias claras de estandarización en la comunicación corporativa a través de sitios web.

Con el paso de los años, Vásquez et al. (2013) han constatado la importancia que se ha dado en Latinoamérica a las NIIF, evidenciado el importante avance en el proceso de convergencia o adopción que han realizado todos los países del estudio (veinte países de Sudamérica y Centroamérica), en especial, aquellos que poseen bolsas de valores más fuertes. Sin embargo, los autores igual evidencian, a través de la revisión de literatura, el bajo uso y calidad de la información en web.

A continuación, identificamos los siguientes usuarios, complementando el concepto de grupos de interés en el contexto de usuarios de información financiera y en general de otras informaciones empresariales, tal como lo detalla el Informe Jenkins (AICPA, 1994):

- Los inversionistas con necesidades de información para las decisiones de la inversión

- Los acreedores con necesidades de información para las decisiones del crédito

- La dirección y los miembros de la junta con necesidades de información para las decisiones sobre manejar el negocio
- Los empleados con necesidades de información para las políticas de compensación y compromisos sobre el beneficio

- Los competidores con necesidades de información para evaluar las fuerzas competitivas, debilidades y estrategia de negocio

- Los reguladores con necesidades de información para evaluar el cumplimiento de las regulaciones

- Los académicos con necesidades de información y datos para investigación

- La prensa con necesidades de información y datos para artículos

- Los usuarios en general con necesidades de información para causas y/o evaluaciones sociales

Frente a la diversidad de grupos de interés o de usuarios de información empresarial, las compañías deben establecer criterios y parámetros que permitan satisfacer diferentes necesidades informativas, incluso considerando que las organizaciones pueden ser lucrativas y no lucrativas.

\section{Revisión de literatura}

Importantes organizaciones relacionadas con la información financiera — como ICAEW (1998), IASC (Lymer et al., 1999), Circa (Trites, 1999), ICAS (1999) o FASB (2000) — han propiciado estudios con el fin de analizar impactos para la profesión y los mercados. A partir de ello, han identificado la necesidad de establecer estándares que permitan a las empresas estructurar la información suministrada a través de Internet. El discussion paper de Lymer et al. (1999) aporta dos definiciones para comprender este fenómeno basado en los usuarios y en el uso tecnológico. Por un lado, define reporting de negocios 
como «la divulgación pública sobre operaciones y datos financieros de un negocio». Por otro, entiende la divulgación basada en la web como «la divulgación pública de datos sobre las operaciones y finanzas de la empresa vía el medio de comunicación, internet» (Lymer et al., 1999).

En los últimos años, hay varios trabajos que analizan el contexto latinoamericano, referido a la divulgación en Internet. No obstante, observamos una concentración de países en diferentes estudios. Los países más estudiados son Argentina, Brasil y Colombia por el cono sur, y México como latino-norteamericano. Han comenzado a aparecer escasos trabajos referidos a Chile. Por su parte, es notoria la ausencia de estudios sobre Perú, Uruguay, Ecuador y Venezuela, cuyos mercados son emergentes en el contexto latinoamericano. Asimismo, se nota un sesgo o interés en investigar empresas cotizadas, mientras que las pymes y las organizaciones del tercer sector permanecen ausentes.

El trabajo de Alali y Romero (2012) realiza un estudio de 84 empresas cotizadas en la Bolsa de Buenos Aires, utilizando una lista de chequeo de 53 variables. $\mathrm{Su}$ investigación no solo busca describir el estado de situación, sino correlacionar diversas variables independientes. El trabajo es interesante, porque, a diferencia de otros, no excluye a empresas del sector financiero y compañías de seguros. Entre los resultados, se observa que las empresas en el sector financiero y de seguros, servicios y minería divulgan más información financiera y no financiera en sus sitios web que las empresas de otras industrias. En el caso del sector minería, estas proporcionan una mayor divulgación sobre la responsabilidad social, lo que da cuenta de un deseo de mejorar la imagen pública que tienen, asociada con el daño al medio ambiente. Alali y Romero (2012), además, verifican empíricamente que las empresas con mayor concentración de la propiedad revelan más información financiera y no financiera que las empresas con menos concentración.

Otra variable que los autores validan y que es relevante mencionar consiste en la rentabilidad y el apalancamiento, los que no tienen un efecto significativo en la divulgación de información en Internet en esta muestra de empresas. Sin embargo, apoyándose en otros autores y tras la medición empírica del reporte de sostenibilidad en mineras peruanas, Hernández (2018) afirma que la influencia de las comunidades campesinas, las reclamaciones de impactos ambientales de poblaciones cercanas a los centros mineros, o el cumplimiento de requerimientos del Estado obligan a una mayor divulgación ambiental y social que justifique y permita legitimar su desempeño.

A partir de su análisis de la situación de 500 compañías colombianas, Gómez y Católico (2009) llegan a la conclusión de que la posición o la situación financiera de las empresas que tienen presencia en Internet mediante un sitio web es significativamente diferente de las compañías que no hacen uso de esta tecnología de la información. Las empresas que sí exponen en Internet sus ingresos operacionales, su activo total, su patrimonio, su utilidad neta y su nivel de endeudamiento son significativamente superiores a aquellas que no adoptan dicha tecnología.

Un trabajo de Morales y Jarne (2009) analiza elementos de divulgación asociados a los conceptos amplios de gobiernos corporativos en una muestra de 33 empresas chilenas, cotizadas y seleccionadas del top índice bursátil (IPSA-40). A través de diferentes dimensiones de la divulgación, evalúan variables independientes para determinar aquellas que ejercen influencias con la divulgación. Como resultado, se ha identificado que las variables tamaño y mercado (ser cotizada en NYSE) sí serían determinantes en esta 
muestra, mientras que endeudamiento, desempeño financiero y tamaño de consejo de administración no influyen significativamente.

Maldonado et al. (2012) realizan un estudio comparado de empresas chilenas y mexicanas, en el cual observan la importancia que dan a la información financiera (reportes e información intermedia). Dicha investigación evidencia una relación negativa entre divulgación y tamaño, lo que podría responder al hecho de que las empresas de menor tamaño requieren de mayor esfuerzo para atraer inversores, por lo cual necesitan mostrar una mayor disposición a la transparencia. Este resultado es diferente a lo confirmado por otros estudios que analizan dichas variables.

Briano y Rodríguez (2012), por su parte, determinan las variables influyentes en la divulgación realizada por 35 empresas españolas y 35 mexicanas. Los autores concluyeron que las variables que tienen influencia significativa son la concentración de la propiedad y la dualidad COB-CEO. Sin embargo, otras variables como el tamaño de la junta y la composición del directorio, el apalancamiento, la rentabilidad (medida en ROA) y tamaño de la empresa no son significativas en el modelo.

Un interesante trabajo comparativo en tres momentos (tres años) fue llevado a cabo por Garay et al. (2013). Los autores examinan 473 empresas latinoamericanas de 6 países (Argentina, Brasil, Chile, Colombia, México y Perú) buscando posibles relaciones entre un índice de revelación basado en las divulgaciones realizadas en Internet y el valor de las empresas de la muestra. Como resultado, confirmaron que industria (sector) y país de origen son variables sensibles y significativas frente a un incremento de índice de divulgación corporativa que trabajan los autores, así como ROA, pero con menor significancia, contrastado a través del test estadístico Q de Tobin. La evidencia contribuye a la literatura, especialmente de países latinoamericanos, sugiriendo que las empresas pueden diferenciarse mediante la adopción de mejoras y la toma de medidas para la divulgación corporativa utilizando Internet.

Finalmente, para cerrar este apartado de revisión de literatura latinoamericana, es importante mencionar los trabajos de Pelayo et al. (2013), y Briano y Rodríguez (2016), quienes estudian empresas cotizadas de los mismos países: Argentina, Brasil, Chile y México. Los primeros, Pelayo et al. (2013), basan su investigación en 135 empresas y reafirman la tendencia del uso de la web para la creciente revelación voluntaria de carácter empresarial y contable no solo por razones de imagen, sino para responder a la creciente demanda de información por inversores, mejorando la calidad y cantidad de informaciones suministradas. El trabajo de los autores presenta un modelo de regresión que determina que la navegabilidad (referido al diseño y usabilidad) es una variable que ejerce influencia significativa sobre la divulgación financiera en Internet en la muestra de empresas estudiadas, mientras que la variable gobiernos corporativos no fue significativa para el modelo.

En todos los países investigados, el fenómeno de la divulgación corporativa vía Internet ya está desarrollándose, con diferentes niveles de profundidad, y aún en materia en desarrollo, con regulaciones incipientes en algunos casos y con mayor asimetría informativa entre empresas latinoamericanas versus los estudios consultados para empresas en países desarrollados.

Aunque no es un trabajo basado en información en Internet, nos parece interesante el tema y tipo de información del trabajo de Hernández (2018), que estudia la realidad de los informes de sostenibilidad 
de empresas cotizadas peruanas. El autor afirma que la mayor apertura a mercados internacionales en la última década ha obligado a las empresas peruanas a ampliar su desempeño con sus distintos grupos de interés, dirigiendo sus acciones y reportes a los inversionistas sociales orientados a establecer mejores relaciones con sus comunidades. Asimismo, apoyándose en otros autores, Hernández explica que, en Perú, el nivel de información de sostenibilidad de las empresas ha aumentado, principalmente, para las empresas con mayor impacto ambiental y social, como es el caso de las empresas mineras y de explotación de petróleo.

Otro trabajo muy interesante sobre empresas peruanas, no dirigido en informaciones en web, pero sí en una materia que pertinente a este artículo, es el de Camacho (2017), que trabaja en el ámbito de la gobernanza corporativa. Este aspecto tiene como principio fundamental la transparencia informativa y, por tanto, se encuentra a un paso de los medios de divulgación. El autor que trabaja basándose en 486 encuestas suministradas por los emisores durante los años 2008-2013 incluye el subíndice «Comunicación y transparencia informativa» entre cuatro índices parciales. Como conclusión, sostiene que, en el caso peruano, la diversidad y la independencia de los miembros del consejo directivo genera un efecto negativo en el rendimiento operativo. Ello puede deberse a que la heterogeneidad del directorio podría causar inconvenientes entre sus miembros, tales como problemas de comunicación, falta de intercambio de opiniones, menos cooperación y conflictos emocionales. Además, puede hacer el proceso de toma de decisiones más lento y menos efectivo.

Cabe señalar que no hemos profundizado en estudios enfocados en otros continentes para darles énfasis a los trabajos latinoamericanos. Sin embargo, es preciso anotar que existe literatura que aborda el fenómeno del uso de los sitios web como elemento comunicador, como lo observamos en trabajos recientes de Saraite et al. (2018), que examina universidades del ranking mundial Academic Ranking of World Universities (ARWU). De acuerdo con sus resultados, las universidades más jóvenes están más interesadas en utilizar la transparencia en la web como estrategia para mejorar su posición competitiva, mientras que las de mayor tamaño, que son las organizaciones más expuestas a la observación pública, estarían más dispuestas a emplear este mecanismo como canal para la rendición de cuentas y mejora de servicios.

También, destacamos el estudio de Khoufi y Khrifech (2018), quienes realizan una investigación empírica correlacional sobre la influencia de diversas variables culturales — como el individualismo, la incertidumbre- y variables institucionales — como desarrollo del país (inflación y educación) y tamaño del mercadosobre los niveles de divulgación en web, basándose en una muestra ajustada de 205 grandes corporaciones cotizadas de diferentes mercados europeos. La regresión mostró que el desarrollo económico del país (significativo y positivo) es el factor determinante de las prácticas divulgación en Internet. De manera similar, el trabajo de Kumar et al. (2017) explora las características y las condiciones de reporting en Internet en 98 compañías cotizadas en Dhaka Stock Exchange (DSE), Bangladech. Los resultados no son alentadores, pues evidenciaron un retraso en el uso de los sitios web de partes de las empresas. Ahmed et al. (2017), por su parte, estudian 172 empresas cotizadas en mercado egipcio (EGX), comparando 2 años (2011 y 2010) siguiendo la misma metodología de otras investigaciones a través de un índice para medir el nivel de divulgación en tres categorías: contenido, soporte al usuario y presentación. A juicio de los autores, las prácticas aún son bajas en comparación con 
sus contrapartes en los países desarrollados. Además, observaron un leve incremento para 2011, lo que se debió a la mayor incertidumbre causada por el levantamiento político a principios de dicho año. Ello habría impulsado las divulgaciones en web de forma permanente y muy probablemente para crecer en el futuro.

En general, países como Argentina, Brasil, Chile y México son los más estudiados en estas materias, lo cual se relaciona con la relevancia de sus bolsas de valores y de su prospección económica, que concentra parte importante de multinacionales latinoamericanas. No obstante, dicha concentración no permite dar una verdadera mirada latinoamericana al tema, puesto que los estudios se enfocan en los mismos países. En ese marco, ampliar el foco a un trabajo comparado de los mercados chilenos y peruanos proporciona evidencia.

\section{Metodología}

El presente estudio consiste en una investigación de tipo empírico, a partir de un análisis exploratorio mediante el que se busca determinar el grado de divulgación de la información corporativa en empresas de los países Chile y Perú. Específicamente, se analizan las empresas utilizadas por Morales y Jarne (2009) del IPSA $^{3}$ de Chile junto con las incluidas del S\&P/BVL Perú General ${ }^{4}$. Para efectos del análisis, se excluirán las empresas que pertenecen al sector bancario y de seguros, en tanto están reguladas de forma particular, por lo cual no pueden compararse con el resto de empresas en la materia investigada. De esta forma, hemos seleccionado un total de 63 empresas (33 de Chile y 30 de Perú).
En esta investigación, la recolección de datos se realizó utilizando una lista de chequeo previamente aplicada en Morales y Jarne (2009). Dicha lista se inspira en un estándar de revisión de recomendaciones y códigos de buenas prácticas realizadas por la Asociación Española de Contabilidad y Administración de Empresas (AECA, 2002) y el International Accounting Standards Committee (IASC, 1999), y permite darle continuidad a una línea de investigación en la materia. En total, trabajaremos con un total de 48 variables dependientes. Estas variables se han agrupado en cinco ítems o dimensiones según características comunes entre sí, los cuales se detallan de la siguiente forma:

\section{Tabla 1. Dimensiones, variables y características}

\begin{tabular}{|l|c|l|}
\hline \multicolumn{1}{|c|}{ Dimensión } & $\begin{array}{c}\mathrm{N}^{\circ} \text { de } \\
\text { variables }\end{array}$ & \multicolumn{1}{|c|}{ Características } \\
\hline $\begin{array}{l}\text { Rendición de } \\
\text { cuentas (RC) }\end{array}$ & 10 & $\begin{array}{l}\text { Aspectos relacionados con } \\
\text { contabilidad, como sus estados } \\
\text { financieros, memoria, entre otros. }\end{array}$ \\
\hline $\begin{array}{l}\text { Compañía y } \\
\text { estrategia (CE) }\end{array}$ & 9 & $\begin{array}{l}\text { Aspectos relacionados con } \\
\text { los antecedentes generales y } \\
\text { estratégicos de las empresas. } \\
\text { Incluye variables que conside- } \\
\text { ran al equipo de gobierno de } \\
\text { la empresa, información sobre } \\
\text { juntas directivas y de accionistas, } \\
\text { carta del presidente, organigrama, } \\
\text { composición accionarial, entre } \\
\text { otros. }\end{array}$ \\
\hline $\begin{array}{l}\text { Análisis finan- } \\
\text { ciero (AF) }\end{array}$ & $\begin{array}{l}\text { Aspectos relacionados con } \\
\text { informaciones que favorezcan la } \\
\text { interpretación y análisis finan- } \\
\text { ciero, ya sea a través de gráficos o } \\
\text { tablas. También, incluye informa- } \\
\text { ción sobre sus acciones o índices } \\
\text { financieros, considerando los } \\
\text { resúmenes de estados contables } \\
\text { relacionados al año actual y años } \\
\text { anteriores. }\end{array}$ \\
\hline
\end{tabular}

3 Es el actual S\&P/CLX IPSA.

4 Dicha información está actualizada hasta el 30 de abril de 2018 


\begin{tabular}{|l|c|l|}
\hline \multicolumn{1}{|c|}{ Dimensión } & $\begin{array}{c}\text { N}^{\circ} \text { de } \\
\text { variables }\end{array}$ & \multicolumn{1}{|c|}{ Características } \\
\hline $\begin{array}{l}\text { Transparencia } \\
\text { (TRAN) }\end{array}$ & 12 & $\begin{array}{l}\text { Disposición en web de diversos } \\
\text { informes -ya sea de riesgo, } \\
\text { medioambiental, social, de cos- } \\
\text { tos-, códigos de ética y de buen } \\
\text { gobierno corporativo. }\end{array}$ \\
\hline $\begin{array}{l}\text { Funcionalidad } \\
\text { (FUNC) }\end{array}$ & 11 & $\begin{array}{l}\text { Es un tanto diferente a las } \\
\text { anteriores, puesto que no hace } \\
\text { referencia a la información que } \\
\text { presenta, sino la forma, ya sea si } \\
\text { permite descargar la información, } \\
\text { si posee buscador interno, si } \\
\text { posee buzón de sugerencias, si da } \\
\text { la posibilidad de recibir alertas de } \\
\text { noticias, información en inglés, } \\
\text { etc. }\end{array}$ \\
\hline
\end{tabular}

Fuente: Elaboración propia

Una vez recolectadas las informaciones según las variables que se hayan establecido, es posible elaborar índices parciales siguiendo el mismo orden y, luego, la agregación de índices parciales nos permite obtener el índice total (IDF-T). Con el índice, ya estaríamos en condiciones de identificar conceptualmente las diferencias existentes entre las divulgaciones por Internet de las empresas estudiadas para poder clasificarlas según la puntuación total del índice y según sus dimensiones.

\section{Desarrollo}

Al interpretar los componentes de la Tabla 2 para los cálculos de medida de tendencia central sobre el índice total, en la revisión de las empresas, con respecto a la media podemos observar un cumplimiento del $64,37 \%$, que está desglosado principalmente por Chile $(74,28 \%)$ siendo el que más se destaca a nivel total con respecto a las dimensiones, encontrándose sobre la media obtenida. Sin embargo, en el índice total, se encuentra Perú $(53,46 \%)$ ubicado bajo la media, lo que demuestra un nivel inferior en la presentación de información corporativa.
Tabla 2. Estadísticas descriptivas: Total de dimensiones

\begin{tabular}{|c|c|c|c|c|c|c|}
\hline País & N $^{\circ}$ & Media & $\begin{array}{c}\text { Desviación } \\
\text { estándar }\end{array}$ & Máx. & Mín. & Rango \\
\hline Chile & 33 & 74,28 & 9,95 & 87,88 & 46,05 & 41,83 \\
\hline Perú & 30 & 53,46 & 17,20 & 74,84 & 5,45 & 69,38 \\
\hline Total & 63 & 64,37 & 17,30 & 87,88 & 5,45 & 82,42 \\
\hline
\end{tabular}

$\mathrm{Al}$ analizar la dispersión se observa que la desviación estándar se mueve entre 9,95\% y 17,20\%. El valor más alto se encuentra en Perú, que está apartado al concentrar el valor más elevado de este estudio.

Se puede afirmar que ni los valores máximos ni los mínimos cuentan con una puntuación perfecta en promedio de las dimensiones. De este modo, se evidencia el nivel de divulgación de las empresas no ha sido perfecto a nivel total. El mayor rango de datos lo demuestra Perú con un 69,38\% en su valor mínimo de $5,45 \%$ a $74,84 \%$.

En el Gráfico 1, presentamos de forma resumida y agrupada por país los resultados por índices que representan todas las dimensiones del estudio para cada uno de los países de la muestra. Se observa claramente el mejor posicionamiento de Chile por encima de Perú en prácticamente todos sus índices. El caso peruano arrojó los índices más bajos y muy por debajo del promedio. El gráfico, también, evidencia las diferencias entre los índices de cada país y entre los países, diferencias que son más relevantes en Chile y Perú y que hemos agrupado bajo el concepto «rendición de cuentas».

Siguiendo a los sectores económicos, las empresas del sector de pesca presentan una mayor tendencia a divulgar información. Sin embargo, los resultados no son concluyentes, puesto que hay también una importante presencia de empresas de elaboración 
de productos y una baja participación del sector de actividades inmobiliarios y servicios sociales (1,05\%). De acuerdo con el índice total calculado para la divulgación de información corporativa (ver Gráfico 2), el sector $^{5}$ con el mejor puntaje es el de pesca $(65,37 \%)$; y el menor puntaje, el sector de extracción de carbón y de lignito $(56,37 \%)$.

\section{Gráfico 1. Índices por dimensión y país}

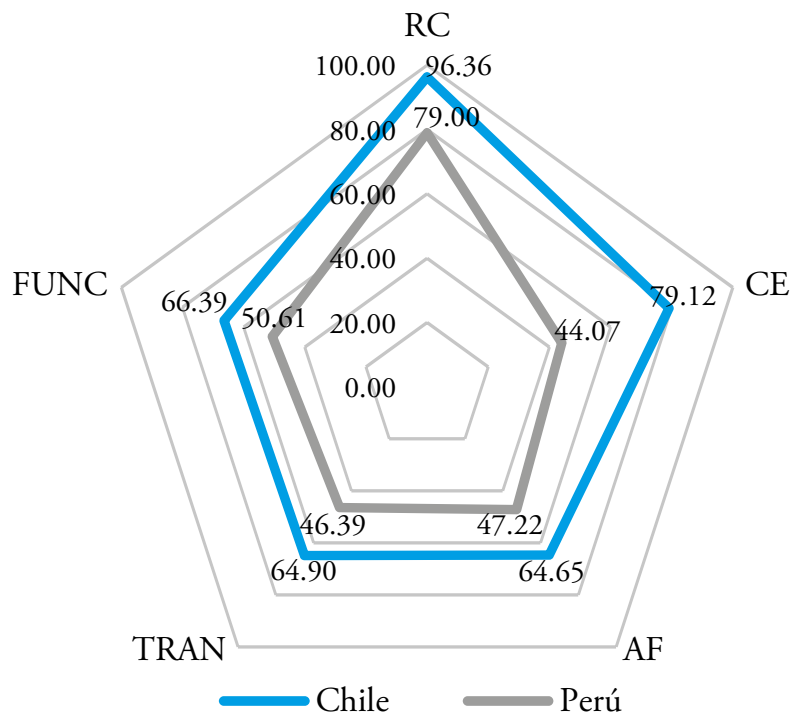

Asimismo, según los criterios establecidos por dimensión, las 3 mejores empresas en presentación de información corporativa a través de sitios webs son Conchatoro $(87,88 \%)$, Latam $(87,47 \%)$ y CTC-A (86,36\%). Mientras, en los peores resultados en la evaluación del cuestionario, se hallan Enel Distribución Perú, empresa Agroindustrial Pomalca y PPX Mining Corp, debido a que, en sus sitios web, divulgan publicidad en lugar de información corporativa.

\section{Gráfico 2. Índice total por sector económico}

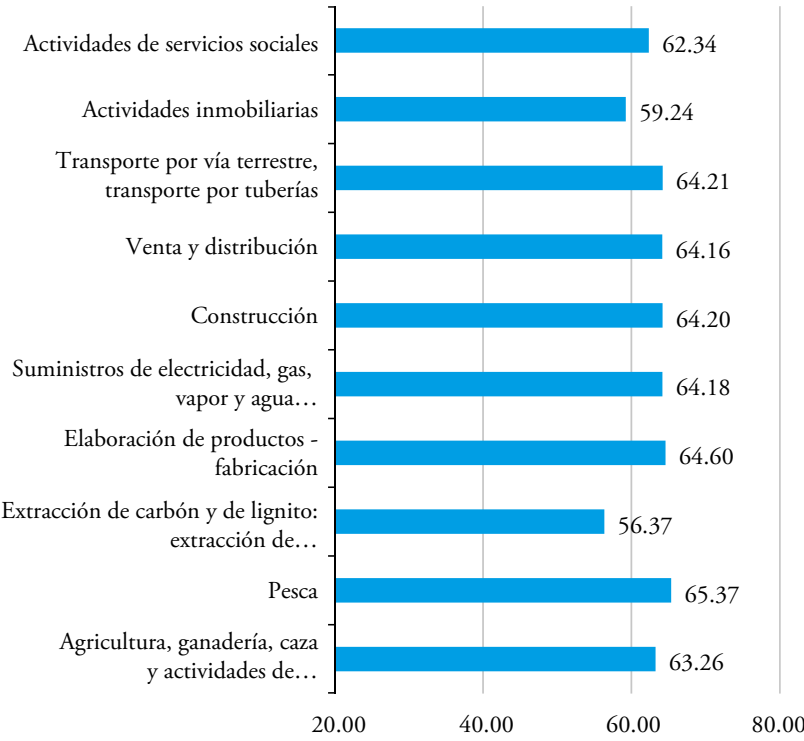

A continuación, analizamos la información obligatoria con respecto a normativa vigente versus voluntaria en sitios web de la muestra de empresas. Como puede verse en la Tabla 4, el cumplimiento de los países con respecto a la información obligatoria asciende a un $74,78 \%$ del total. En este índice, los valores de Chile se encuentran por encima del promedio obtenido $(87,88 \%)$, mientras que Perú se encuentra por debajo de la media obtenida (60,37\%). Sin embargo, los resultados no son favorables, en la medida que es un requerimiento obligatorio en divulgación de información. En el caso del índice voluntario, el promedio de las empresas asciende a 63,48\%. En este marco, Chile se encuentra sobre la media obtenida $(68,32 \%)$, mientras que nuevamente solo Perú se encuentra alejado de esta con un 52,31\%.

5 El número de empresas por sector fueron tres en Agricultura, ganadería, caza y actividades de servicio conexas; dos en Pesca; trece en Extracción de carbón y de lignito: extracción de turba; diecisiete en Elaboración de productos - fabricación; once en Suministros de electricidad, gas, vapor y agua caliente; dos en Construcción; ocho en Venta y distribución; cinco en Transporte por vía terrestre, transporte por tuberías; una en Actividades inmobiliarias; y una en Actividades de servicios sociales. 
Tabla 4. Comparación de la media por índice de información obligatoria versus voluntaria

\begin{tabular}{|c|c|c|c|}
\hline País & $\begin{array}{c}\text { Información } \\
\text { obligatoria }\end{array}$ & $\begin{array}{c}\text { Información } \\
\text { voluntaria }\end{array}$ & Diferencia \\
\hline Perú & 60,37 & 52,31 & 8,06 \\
\hline Chile & 87,88 & 68,32 & 19,56 \\
\hline Total & 74,78 & 60,69 & 14,09 \\
\hline
\end{tabular}

Por lo tanto, se puede afirmar que las empresas divulgan su información, porque la ley lo exige y no por un tema voluntario de complacer a los usuarios. Estos datos se pueden observar de una forma más detallada, a partir de los resultados por índice y país, en la Tabla 5.

Tabla 5. Comparación de la media por índice de información obligatoria versus voluntaria

\begin{tabular}{|c|c|c|c|c|c|c|}
\hline Pais & Media & Mediana & $\begin{array}{c}\text { Desviación } \\
\text { estándar }\end{array}$ & Máx. & Mín. & Rango \\
\hline \multicolumn{7}{|c|}{ Rendición de cuentas } \\
\hline Chile & 96,36 & 100,00 & 5,49 & 100,00 & 80,00 & 20,00 \\
\hline Perú & 79,00 & 80,00 & 23,83 & 100,00 & 0,00 & 100,00 \\
\hline Total & 88,10 & 90,00 & 18,91 & 100,00 & 0,00 & 100,00 \\
\hline \multicolumn{7}{|c|}{ Compańía y estrategia } \\
\hline Chile & 79,12 & 77,78 & 14,37 & 100,00 & 55,56 & 44,44 \\
\hline Perú & 44,07 & 44,44 & 20,73 & 77,78 & 0,00 & 77,78 \\
\hline Total & 62,43 & 66,67 & 24,88 & 100,00 & 0,00 & 100,00 \\
\hline \multicolumn{7}{|c|}{ Análisis financiero } \\
\hline Chile & 64,65 & 66,67 & 22,73 & 100,00 & 16,67 & 83,33 \\
\hline \multicolumn{7}{|c|}{ Transparencia } \\
\hline Chile & 64,90 & 66,67 & 12,63 & 83,33 & 41,67 & 41,67 \\
\hline Perú & 46,39 & 50,00 & 21,07 & 75,00 & 0,00 & 75,00 \\
\hline Total & 56,08 & 58,33 & 19,41 & 83,33 & 0,00 & 83,33 \\
\hline \multicolumn{7}{|c|}{ Funcionalidad } \\
\hline Chile & 66,39 & 72,73 & 18,11 & 100,00 & 27,27 & 72,73 \\
\hline Perú & 50,61 & 54,55 & 12,33 & 72,73 & 27,27 & 45,45 \\
\hline Total & 58,87 & 63,64 & 17,42 & 100,00 & 27,27 & 72,73 \\
\hline
\end{tabular}

En la tabla anterior, se puede apreciar las diferencias en los promedios, las desviaciones estándar y los rangos para los datos de la muestra, siempre comparativamente. De esta manera, tiene sentido lo indicado en párrafos anterior: existen diferencias entre ambas muestras y las empresas chilenas estarían logrando mejores resultados en la divulgación en web por encima de sus pares peruanas. Datos aún más desagregados y las variables evaluadas en esta medición se pueden ver en anexo al final del artículo.

Como una forma de verificar si las diferencias detectadas en el nivel de divulgación entre las empresas de ambos países son significativas, aplicaremos algunas pruebas estadísticas para contrastar la hipótesis de igualdad de medias entre los grupos. Este trabajo lo centraremos en comprobar tres hipótesis nulas, para lo cual utilizamos el test de Wilcoxon, válido para datos no paramétricos bajo muestras independientes, las que presentaremos a continuación

- Hipótesis $1\left(H_{1}\right)$ : El índice de divulgación legal y el índice de divulgación voluntario no presenta diferencias significativas entre ambos países.

- Hipótesis $2\left(\mathrm{H}_{2}\right)$ : El índice de divulgación legal de las empresas peruanas y chilenas no presenta diferencias significativas entre ambos países.

- Hipótesis $3\left(H_{3}\right)$ : El índice de divulgación voluntario de las empresas peruanas y chilenas no presenta diferencias significativas entre ambos países.

Aplicados los test de Wilcoxon, encontramos para $H_{1}$ una suma de rangos de 4971,5 y un valor de significancia de 0,00000216159. A partir de ello, podemos rechazar la igualdad de medias entre los índices y afirmar que existen diferencias significativas entre ambos países, medidas en todas las empresas con sus dos índices, siempre comparando la muestra de empresas chilenas versus peruanas. 
Para $H_{2}$, con una suma de rangos de 587,5 y un p-valor de 0,000000295394, podemos inferir al 5\% de significancia. Así, obtenemos evidencia suficiente para rechazar la hipótesis nula de igualdad de los índices de divulgación legal entre las empresas chilenas y peruanas: las empresas chilenas mostraron un mayor índice de revelación legal que sus similares peruanas. Otro aspecto a destacar es la homogeneidad de la información de las empresas para el índice analizado. Los índices de las empresas chilenas presentan menor homogeneidad, lo que se refleja en la desviación estándar 7,15979. En cambio, en el caso de las empresas peruanas, la desviación estándar es superior - asciende a 24,0057-, lo cual representa una mayor diversidad.

Finalmente, $H_{3}$ arrojó una suma de rangos de 647 y un valor de significancia de 0,0000165095 al igual que el anterior, notoriamente inferior al 5\% utilizado en este test. A partir de ello, como el valor $\mathrm{p}$ es menor que el $5 \%$, hay evidencia suficiente para rechazar la hipótesis nula de igualdad de los índices de divulgación voluntario entre las empresas chilenas y peruanas. En este marco, el promedio mayor del índice corresponde al caso chileno. $\mathrm{Al}$ analizar la homogeneidad en este índice, las empresas chilenas y las peruanas presentan desviación estándar similares, de 12,38 y de 15,24 respectivamente.

\section{Conclusiones}

El desarrollo de Internet como un medio para divulgar la información corporativa ha creado una nueva forma de comunicación corporativa entre la empresa y sus grupos de interés. En esta investigación, se ofrece una contribución al conocimiento sobre la dinámica divulgativa de la información corporativa que realizan las empresas cotizadas de Chile y Perú.

Debido a lo anterior, se realizó el presente estudio, que ha tenido como propósito principal establecer qué tipo de información revelan y divulgan las empresas en sus sitios webs. Una vez verificadas las páginas web de las 63 empresas objeto de estudio, se pudo inferir que estas revelan en mayor proporción información referente a su situación financiera, es decir, todo lo referente a sus estados financieros, notas y dictámenes, así como aquella asociada a su portafolio de productos y servicios. Esto se debe a que las compañías, en su mayoría, consideran Internet como una ventana para publicitar lo que ofrecen, pensando atraer cada vez mayor número de clientes y mejorar resultados, lo que se observó en la mayoría de las empresas peruanas. No obstante, en un mundo globalizado como el actual, se debe dar muestra de otro tipo de aspectos relevantes, como lo son el compromiso con la sociedad y el ambiente, así como el cumplimiento de las buenas prácticas empresariales y un mayor acceso a la información para poder atraer mayor inversión de capital tanto nacional como extranjero en pro del crecimiento y sostenibilidad de dichas compañías.

Asimismo, los resultados descriptivos muestran que las sociedades cotizadas en Chile obtienen un índice total del $74,28 \%$, mientras que, con una diferencia significativa, el índice de Perú alcanza el 53,46\%. Estos países han concentrado en mayor medida sus esfuerzos a la divulgación de información contable-financiera. Aun así, Chile destaca superando a Perú en los cinco índices parciales. De hecho, el caso peruano es el más disminuido en los resultados del cuestionario aplicado: sus índices son los más bajos y quedan por debajo del promedio. Ello lleva a pensar que aún falta una mayor cultura de la divulgación en empresas, que debiera ser acompañada de una mayor regulación al respecto.

Las causas de lo anterior deberían ser objeto de otra investigación, pero la literatura ha comprobado que mercados más internacionales, tamaño de las compañías y multinacionalización de sus empresas tienden a estar relacionados con mayores volúmenes de divulgación 
en Internet. A pesar de que, en este estudio, no lo hemos comprobado, podría haber algo de ello revisando algunos datos de las bolsas de ambos países, debido a que las compañías de la muestra utilizada se corresponden con las principales del índice bursátil de cada país, lo que se condice con aspectos de tamaño y multinacionalización.

Se ha comprobado que las empresas utilizan Internet principalmente para difundir información financiera tradicional. Sin embargo, no logran explotar el potencial de esta plataforma para mejorar otros aspectos, tales como los referentes a la información no financiera. Ello sugiere que las compañías no han implementado metodologías de medición ni revelación basadas fielmente en las RSC, apuntando a los indicadores ESG (Enviromental, Social and Governance). Como consecuencia, desperdician experiencia y métodos ya aceptados de la contabilidad social y extensibles a otras materias de interés público.

\section{Referencias bibliográficas}

Ahmed, A., Burton, B. \& Dunne, T. (2017). The determinants of corporate internet reporting in Egypt: An exploratory analysis. Journal of Accounting in Emerging Economies, 7(1), 35-60. https://doi.org/10.1108/ JAEE-04-2015-0024

Alali, F. \& Romero, S. (2012) The use of the internet for corporate reporting in the Mercosur (Southern common market): The Argentina case. Advances in Accounting, Incorporating Advances in International Accounting, (28), 157-167. https://doi.org/10.1016/j.adiac.2012.03.009

American Institute of Certified Public Accountants [Aicpa] (1994). Improving Business Reporting. A Customer Focus: Meeting the information needs of investors and creditors" (comprehensive report of the Special Committee on Financial Reporting). Recuperado de https://www. aicpa.org/InterestAreas/FRC/AccountingFinancial Reporting/DownloadableDocuments/Jenkins\%20 Committee\%20Report.pdf
Asociación Española de Contabilidad y Administración de Empresas [AECA] (2002). Código de buenas prácticas para la divulgación de información financiera en Internet. Madrid: AECA.

Asociación Española de Contabilidad y Administración de Empresas [AECA] (2004). Certificación y auditoría de la información digital. Madrid: AECA.

Briano, G. y Rodríguez, L. (2012). Corporate information transparency on the internet by listed companies in Spain (IBEX 35) and Mexico (IPYC). The International Journal of Digital Research, (12), 1-37. https://doi. org/10.4192/1577-8517-v12_1

Briano, G. y Rodríguez, L. (2016). Corporate governance ratings on listed companies: An institutional perspective in Latin America. European Journal of Management and Business Economics, 25(2), 63-75. https://doi. org/10.1016/j.redeen.2016.01.001

Camacho, N. (2017). Efecto de la aplicación de las buenas prácticas de gobierno corporativo en el desempeńo operativo de las empresas peruanas listadas en la Bolsa de Valores de Lima para los ańos 2008-2013 (tesis de licenciatura). Universidad de Piura, Piura, Perú.

Financial Accounting Standard Board [FASB] (2000). Business reporting research project, electronic distribution of business reporting information (Steering Committee Report). Recuperado de http://www.fasb.org/brrp/ brrp_main.shtml

Financial Accounting Standard Board [FASB] (2001). Business and financial reporting, challenges from the new economy (Special Report, No 219-A). Recuperado de http://www.fasb.org/new_economy.shtml

Gandía, J. (2001). La divulgación de información en la era digital. Madrid: AECA.

Gandía, J. y Pérez, T. (2005). e-Gobierno Corporativo y transparencia en las sociedades cotizadas españolas. Un estudio empirico. Madrid: Editorial CNMV.

Garay, U., González, M., Guzmán, A. y Trujillo, M. (2013). Internet-based Corporate disclosure and market value: 
Evidence from Latin America. Emerging Markets Review, 17, 150-168. https://doi.org/10.1016/j.ememar.2013.09.002

Giner, B. (1995). La divulgación de información financiera: Una investigación empirica. Madrid: Instituto de Contabilidad y Auditoría de Cuentas.

Gómez, E. \& Católico, D. (2009) Revelación y divulgación de la información financiera y no financiera on-line de las 500 empresas más representativas en Colombia. Cuadernos de Contabilidad, 10(27), 269-318.

Hernández, J. (2018). Influencia de la naturaleza internacional de empresas peruanas en su información de sostenibilidad. Revista de Comunicación, 17(1), 74-9. https://doi.org/10.26441/RC17.1-2018-A4

Institute of Chartered Accountants in England and Wales [ICAEW] (1998). The $21^{\text {st }}$ Century Annual Report. Londres. Recuperado de https://www.icaew.com/-/media/ corporate/files/technical/research-and-academics/ publications-and-projects/financial-reporting-publications/the-21st-century-annual-report.ashx

Institute of Chartered Accountants Scotland [ICAS] (1999). Business reporting: The inevitable change? Discussion document. Glasgow: Institute Chartered Accountants Sclotland.

Khoufi, W. \& Khrifech, D. (2018). Country-specific characteristics influencing websites based information disclosure practices. Accounting and Management Information Systems, 17(3), 374-405. https://doi.org/ 10.24818/jamis.2018.03004

Kumar, R., Mahboob, D. \& Mehadi, M. (2017) Web-based corporate reporting: An exploratory study on the Bangladechi companies. Middle East Journal of Business 12(3), 25-30. https://doi.org/10.5742/MEJB.2017.92972

Lymer, A., Debrency, R., Gray, G. \& Rahman, A. (1999). Business reporting on the Internet (discussion paper). International Accounting Standard Committee [IASC], London.

Maldonado, S., Escobar, N. \& Marín, M. (2012). Divulgación de información contable-financiera en el sitio web de empresas cotizadas de Chile y México: Un estudio exploratorio. Cuadernos de Contabilidad, 13(33), 395-431.

Morales, F. \& Jarne, J. (2006) Clasificación internacional de los sistemas contables de Argentina, Brasil y Chile. Panorama Socioeconómico, (32), 90-95.

Morales, F. \& Jarne, J. (2009). e-Gobierno corporativo: evidencia en Chile sobre la divulgación de información en internet. Capic Review, 7, 45-62.

Pablo, R. (2006) Internet y la nueva economía en las empresas. Estudio del caso Amadeus. Madrid: Institutos de Estudios Económicos.

Pelayo, M., Fuertes, Y., Cuellar, B. \& Arias, M. (2013) Impacto de la divulgación de información financiera en internet de las empresas en América Latina. Global Conference on Business and Finance Proceedings 8(1), 772-781.

Saraite, L., Rodríguez, M. \& de Rosario, A. (2018). Exploring determining factors of web transparency in the world's top universities. Revista de Contabilidad, 21(1), 63-72. https://doi.org/10.1016/j.rcsar.2017.07.004

Serrano, C., Fuertes, Y. \& Chaparro, F. (2003) ¿Por qué las empresas revelan información financiera en Internet? Dos estudios empíricos. Jornadas Contabilidad y Empresa Digital. Asociación Española de Profesores Universitarios de Contabilidad, Huesca.

Trites, G. (1999). The impact of technology on financial and business reporting. Toronto: Chartered Accountants of Canada.

Vásquez, N., Carril, M. \& Pascual, M. (2013) Estado actual de Latinoamérica en relación a su proceso de adopción de las NIIF. Revista Contabilidad y Auditoría Investigaciones en Teoria Contable, (38), 55-104.

Fecha de recepción: 08 de marzo de 2019 Fecha de aceptación: 23 de mayo de 2019 Correspondencia: fmorales@ubiobio.cl rholland@ubiobio.cl camvarel@alumnos.ubiobio.cl 
Anexo. Detalle de variables evaluadas y cumplimiento por país

\begin{tabular}{|c|c|c|c|}
\hline No. & Dimensión rendición de cuentas & Chile & Perú \\
\hline $\mathrm{V} 1$ & Presenta balance general último ejercicio. & 100,00 & 93,33 \\
\hline $\mathrm{V} 2$ & Presenta estado de cambios patrimonio último ejercicio & 100,00 & 90,00 \\
\hline $\mathrm{V} 3$ & Presenta estado de situación financiera último ejercicio & 100,00 & 93,33 \\
\hline V4 & Presenta estado de flujos de efectivo último ejercicio & 100,00 & 93,33 \\
\hline V5 & Presenta notas explicativas a los estados financieros último ejercicio & 100,00 & 86,67 \\
\hline V6 & Presenta memoria anual último ejercicio & 96,97 & 76,67 \\
\hline V7 & Presenta información histórica anual & 100,00 & 93,33 \\
\hline V8 & Presenta información intermedia/interina & 100,00 & 83,33 \\
\hline V9 & Presenta resultados financieros (cuentas de pérdidas y ganancias) de forma segmentada & 93,94 & 72,73 \\
\hline V10 & $\begin{array}{l}\text { Otras informaciones de la compañía sobre segmentos de negocios o unidades operativas (solo descriptivo } \\
\text { en este caso) }\end{array}$ & 16,67 & 63,33 \\
\hline No. & Dimensión compañía y estrategia & Chile & Perú \\
\hline $\mathrm{V} 1$ & Posee carta del presidente de la compañía (como documento independiente o en la memoria). & 96,97 & 63,33 \\
\hline $\mathrm{V} 2$ & Divulga equipo de gobierno de la empresa. & 100,00 & 90,00 \\
\hline V3 & Divulga organigrama de la empresa. & 84,85 & 13,33 \\
\hline V4 & Divulga información sobre juntas directivas. & 42,42 & 6,67 \\
\hline V5 & Divulga composición accionarial. & 87,88 & 63,33 \\
\hline V6 & Divulga información sobre asambleas de accionistas. & 84,85 & 33,33 \\
\hline V7 & Divulga información sobre pagos de dividendos (fecha de pago, monto de dividendo por acción). & 63,64 & 20,00 \\
\hline V8 & $\begin{array}{l}\text { Divulga explicación y figura de la estructura empresarial (si tuviese empresas relacionadas, dominante o } \\
\text { controlada). }\end{array}$ & 93,94 & 33,33 \\
\hline V9 & Presenta alguna información sobre estrategias de negocios o empresarial. & 57,58 & 73,33 \\
\hline No. & Dimensión análisis financiero & Chile & Perú \\
\hline $\mathrm{V} 1$ & Divulga tablas resúmenes de estados contables del ejercicio actual. & 63,64 & 33,33 \\
\hline V2 & Divulga tablas resúmenes de estados contables de ejercicios anteriores. & 51,52 & 36,67 \\
\hline $\mathrm{V} 3$ & Proporciona ratios financieros. & 93,94 & 46,67 \\
\hline V4 & Divulga gráficos y/o cuadros explicativos de situación financiera. & 66,67 & 70,00 \\
\hline V5 & $\begin{array}{l}\text { Divulga información sobre ranking de la empresa en clasificaciones (clasificadoras de riesgos, y/o premios, } \\
\text { reconocimientos, ranking empresariales). }\end{array}$ & 36,36 & 56,67 \\
\hline V6 & Divulga información online de bolsa o precio de la acción en tiempo real. & 75,76 & 40,00 \\
\hline
\end{tabular}




\begin{tabular}{|c|c|c|c|}
\hline No. & Dimensión transparencia & Chile & Perú \\
\hline V1 & Posee código de buen gobierno (o prácticas corporativas). & 81,82 & 26,67 \\
\hline $\mathrm{V} 2$ & Posee información sobre buen gobierno (o prácticas corporativas). & 75,76 & 43,33 \\
\hline V3 & Posee código de ética. & 84,85 & 80,00 \\
\hline V4 & Posee informe auditor de auditor externo. & 93,94 & 66,67 \\
\hline V5 & Posee informes sobre riesgos. & 100,00 & 16,67 \\
\hline V6 & Posee informe medioambiental (GRI u otro). & 66,67 & 73,33 \\
\hline V7 & Posee informe social. & 66,67 & 73,33 \\
\hline V8 & Posee informe sobre capital intelectual. & 3,03 & 3,33 \\
\hline V9 & Posee informe de costes. & 3,03 & 33,33 \\
\hline V10 & Posee informe de gestión. & 100,00 & 76,67 \\
\hline V11 & Posee previsiones o informes proyectados. & 27,27 & 3,33 \\
\hline V12 & Posee información sobre conferencias y presentaciones en Power Point, video o audio. & 75,76 & 60,00 \\
\hline No. & Dimensión funcionalidad & Chile & Perú \\
\hline V1 & El sitio web permite descargas. & 96,97 & 100,00 \\
\hline $\mathrm{V} 2$ & Posee buscador interno el sitio. & 78,79 & 53,33 \\
\hline V3 & Posee buzón de sugerencias. & 33,33 & 46,67 \\
\hline V4 & Dispone e-mail para consultas. & 84,85 & 80,00 \\
\hline V5 & Posee un espacio de preguntas (FAQ, por sus siglas en inglés). & 51,52 & 36,67 \\
\hline V6 & Posee un espacio de novedades o noticias actualizado. & 75,76 & 70,00 \\
\hline V7 & Ofrece la posibilidad de recibir alertas, newsletters al e-mail. & 45,45 & 46,67 \\
\hline V8 & El sitio web posee información financiera en inglés. & 54,55 & 6,67 \\
\hline V9 & El sitio web posee información empresarial (cualitativa) en inglés. & 69,70 & 10,00 \\
\hline V10 & El sitio web se presenta en más de un idioma (idioma del país y otro). & 75,76 & 73,33 \\
\hline V11 & Posee vínculos a otros sitios (reguladores, auditores, filiales, etc.). & 63,64 & 33,33 \\
\hline
\end{tabular}

